\title{
CCD (Charge Coupled Device) funcionamento e sua aplicação em sensoriamento remoto
}

\author{
LUCIANO FARINHA WATZLAWICK \\ Pedro Roberto de Azambuja Madruga \\ Rudiney SoARES PEREIRA
}

\section{RESUMO}

O presente artigo aborda conceituações, comentários sobre a composição, método de funcionamento, vantagens e desvantagens do uso de CCD, bem como sua aplicação em sensoriamento remoto, para tanto discorre-se sobre os fundamentos físicos do sensoriamento remoto, espectro eletromagnético, propagação da radiação eletromagnética, interações entre a energia e a atmosfera, janelas atmosféricas e o sistema de aquisição de informações (detecção, registro e armazenamento).

Palavras-chave: CCD (Charge Coupled Device), Sensoriamento Remoto 


\section{ABSTRACT}

The present article concerns about concepts, comments on the composition, working method, advantages and disadvantages of using the CCD, and its application in remote sensing as well, and to do so, we discuss the physical principles of the remote sensing, electromagnetic spectrum, propagation of the electromagnetic radiation, interactions between energy and atmosphere, atmospheric windows and the information acquisition system (detection, record and storage).

Key words: CCD (Charge Coupled Device), Remote Sensing

\section{INTRODUÇÃO}

A utilização de imagens para estudos de monitoramento ambiental, bem como de mapeamentos está evoluindo cada vez mais, primeiramente devido a resolução espacial dos atuais sensores ainda não possuem uma resolução temporal adequada para determinados levantamentos, ou os custos para aquisição das mesmas são altos, ou devido as condições climáticas muitas vezes não serem adequadas, inviabilizando muitas vezes sua aquisição. Com a operacionalização de câmaras que utilizam o CCD como elemento sensível para registro de imagens, este empecilho para a aquisição de imagens para fins de sensoriamento remoto poderá ser solucionado, devido poder-se assim realizar as tomadas das imagens no momento adequado, bem como com resolução apropriada para tal finalidade em função do tipo de câmara utilizada.

\section{DESENVOLVIMENTO}

\subsection{Fundamentos físicos do sensoriamento remoto}

A energia captada pelos sensores pode ser oriunda de uma fonte externa ao alvo (sol), interna (energia térmica própria dos alvos), ou ainda proveniente do próprio sensor (pulsos de microondas). A energia disponível na terra quase que na sua totalidade tem sua origem no sol, de onde se propaga para todo o espaço na forma de radiação eletromagnética, deslocando-se na forma de ondas, com uma velocidade de $300.000 \mathrm{Km} / \mathrm{s}$. Como qualquer fenômeno ondulatório, os diferentes tipos de radiação eletromagnética podem ser caracterizados pelo seu comprimento de onda e sua freqüência, os quais se relacionam com a velocidade de propagação por: 


$$
C=\lambda \cdot v
$$

Onde:

$\mathrm{C}=$ velocidade $\mathrm{em} \mathrm{m} / \mathrm{s}$

$\lambda=$ comprimento de onda em $\mathrm{m}$

$v=$ freqüência em Hertz

Conforme CROSTA \& SOUZA FILHO (1997), a radiação eletromagnética utilizada em sensoriamento remoto abrange diferentes partes do espectro eletromagnético, o qual apesar de representar quase um contínuo, foi dividido em regiões com denominações específicas em função das fontes e tipos de processos envolvidos na geração de radiação. Os diferentes tipos de radiação eletromagnética podem ser divididos de acordo com seu comprimento de onda $(\lambda)$, geralmente medidos em microns $(\mu \mathrm{m})\left(10^{-6} \mathrm{~m}\right)$, ou de acordo com sua freqüência $(v)$.

Existe no espectro eletromagnético os diferentes tipos de radiação: radiação gama, raios-x, ultravioleta, visivel, infravermelho, microondas, TV/rádio.

A radiação gama $\left(10^{-8}\right.$ a $\left.10^{-5} \mu \mathrm{m}\right)$, possui uma alta energia emitida por materiais radioativos, muito utilizada na medicina (radioterapia) e radiografia industrial.

Os raios-x $\left(10^{-5}\right.$ a $\left.10^{-3} \mu \mathrm{m}\right)$ são produzidos pelo freamento de elétrons de alta energia, seu médio poder de penetração a torna adequada para aplicações na medicina (radiografia) e nas técnicas de controle de qualidade.

A radiação ultravioleta $\left(10^{-3}\right.$ a $\left.10^{-1} \mu \mathrm{m}\right)$ é uma radiação emitida em grande quantidade pelo sol, entretanto é bastante atenuada pela camada de ozônio atmosférico antes de atingir a superfície terrestre.

O conjunto das radiações que podem ser percebidas pelo sistema visual humano é denominado de visível $(0,4$ a $0,7 \mu \mathrm{m})$ ou luz. A cor é uma sensação produzida pelo estímulo luminoso e as diferentes componentes do espectro visível produzem sensações de cor que dependem de seu comprimento de onda (ou freqüência).

A região espectral do infravermelho esta subdividida em três sub-regiões: infravermelho próximo $(0,7$ a $1,3 \mu \mathrm{m})$, infravermelho médio $(1,3$ a $3,0 \mu \mathrm{m})$ e infravermelho distante $(3,0 \mathrm{a}<1 \mathrm{~mm}) . \mathrm{Na}$ superfície terrestre, as radiações do infravermelho próximo podem ser encontradas em quantidades bastante significativas na radiação solar enquanto que as radiações do infravermelho médio e distante, são conhecidas como radiação termal e tem sua fonte mais impor- 
tante nos objetos terrestres (mesmo na temperatura ambiente).

Em sensoriamento remoto as radiações de microondas $\left(10^{3}\right.$ a $\left.10^{5} \mu \mathrm{m}\right)$ são utilizadas nos sistemas de RADAR e são importantes pela sua capacidade de gerar imagens através das nuvens.

As radiações eletromagnéticas de freqüência menor que $300 \mathrm{MHz}$ são utilizadas principalmente em telecomunicações, denominando-se de TV/rádio $\left(10^{5} \mathrm{a} 10^{8} \mu \mathrm{m}\right)$.

Segundo a lei da Teoria Ondulatória, a radiação se propaga na forma de onda, à velocidade da luz, segundo um campo elétrico $(E)$ e um campo magnético $(M)$, ortogonais entre si, de modo harmônico e com uma trajetória senoidal.

A teoria de Max Planck ou Teoria Ondulatória, afirma que a energia ou luz é composta por unidades discretas denominadas de fótons ou quanta, sendo a energia de um fóton inversamente proporcional ao seu comprimento de onda. A intensidade de radiação (brilho da luz visível), é em função do número de fótons ou da amplitude da onda propagante, expressas da seguinte maneira:

$$
Q=h . v \text { ou } Q=h . c / \lambda
$$

Onde:

$$
\begin{aligned}
& Q=\text { energia de um fóton em Joules } \\
& h=\text { constante de Planck }(6,626 \times 10-34 \mathrm{~J} / \mathrm{seg}) \\
& v=\text { freqüência } \\
& C=\text { velocidade da luz } \\
& \lambda=\text { comprimento de onda }
\end{aligned}
$$

O sensoriamento remoto é um sistema de aquisição de informações, podendo ser dividido em um sistema de coleta de dados e um sistema de análise de dados. O sistema de coleta de dados possui duas funções: detecção da radiação proveniente da superfície e transformação da radiação, para posterior transmissão e registro.

A detecção da radiação depende de certos requisitos: primeiro deve haver uma fonte de radiação eletromagnética (Sol), essa radiação deve se propagar pela atmosfera (Ar), até atingir a superfície terrestre (Alvos). Ao atingir a superfície terrestre sofrerá interações, produzindo uma radiação de retorno, que se propagará pela atmosfera (Ar), ou pelo meio físico entre os alvos e o sensor (Câmara de Vídeo, Fotografia...). O que chega até o sensor é uma certa intensidade de energia eletromagnética (radiação), que será transformada em sinal e armazenada (Fitas, Filmes...). 
A radiação eletromagnética antes de ser captada pelos sensores atravessa a atmosfera, sofrendo alterações. As principais interações que ocorrem entre a energia e a atmosfera são o espalhamento (difusão da energia pelas partículas atmosféricas) e absorção (parte da energia é absorvida pelos gases atmosféricos, como vapor de água, $\mathrm{CO}_{2}$ e ozônio), em ambos os casos uma parcela de energia é perdida, sendo que a parcela remanescente sofre modificações antes de ser captada pelo sensor.

Quando a direção da energia é modificada de forma aleatória, devido a presença de partículas de gases e aerossóis na atmosfera, ocorre o chamado espalhamento atmosférico, resultando na diminuição da qualidade das imagens. Os efeitos mais intensos ocorrem nos comprimentos de onda do visível e do infravermelho próximo, existem três tipos de espalhamento, conforme descritos por CROSTA \& SOUZA FILHO (1997): Rayleigh, Mie e Não-Seletivo.

O espalhamento Rayleigh é o mais comum e ocorre quando a energia incidente encontra partículas de tamanho inferior ao comprimento de onda do fóton, sendo este espalhamento responsável pela cor azul do céu, significando que a componente azul da energia solar sofre um espalhamento maior do que as demais componentes, devido ao seu pequeno comprimento de onda. Por outro lado, o espalhamento Mie, ocorre quando o comprimento de onda da luz incidente é equivalente ao tamanho das partículas, sendo os principais causadores deste espalhamento o vapor d'água, fumaça e a poeira, afetam os comprimentos de onda maiores que o azul.

Afetando todos os comprimentos de onda da mesma forma, o espalhamento não-seletivo ocorre quando as partículas atmosféricas são maiores do que o comprimento de onda da luz incidente, dando uma cor esbranquiçada à imagem. Nuvens, neblina e gotas d'água, todas formadas por partículas com diâmetros superiores aos comprimentos de onda que estão sendo cobertos pelos atuais sensores.

Os principais gases responsáveis pela absorção da radiação eletromagnética são: vapor d'água, ozônio, dióxido de carbono e metano, o oxigênio e hidrogênio, apesar de serem os mais abundantes, absorvem relativamente menor quantidade de energia. Esta absorção faz com que a aquisição de informações para sensoriamento remoto fique restrita a algumas porções do espectro eletromagnético, devido haver regiões onde a energia é totalmente absorvida.

Na região do raio- $x$ e raio- $\gamma$, abaixo de $0,3 \mu \mathrm{m}$, toda a 
energia é absorvida pela camada de ozônio da atmosfera superior, também sendo bastante afetada pela absorção atmosférica a região do ultravioleta. Porém, as regiões do visível e do infravermelho possuem uma alta transmitância, sendo denominadas de janelas atmosféricas onde o sensoriamento remoto se concentra para aquisição de informações.

Os intervalos de baixa absorção são denominados de bandas de absorção atmosférica. A Figura 1, mostra as principais janelas atmosféricas (espaços claros) e bandas de absorção (espaços escuros), onde cada um dos componentes atmosféricos absorvem energia.

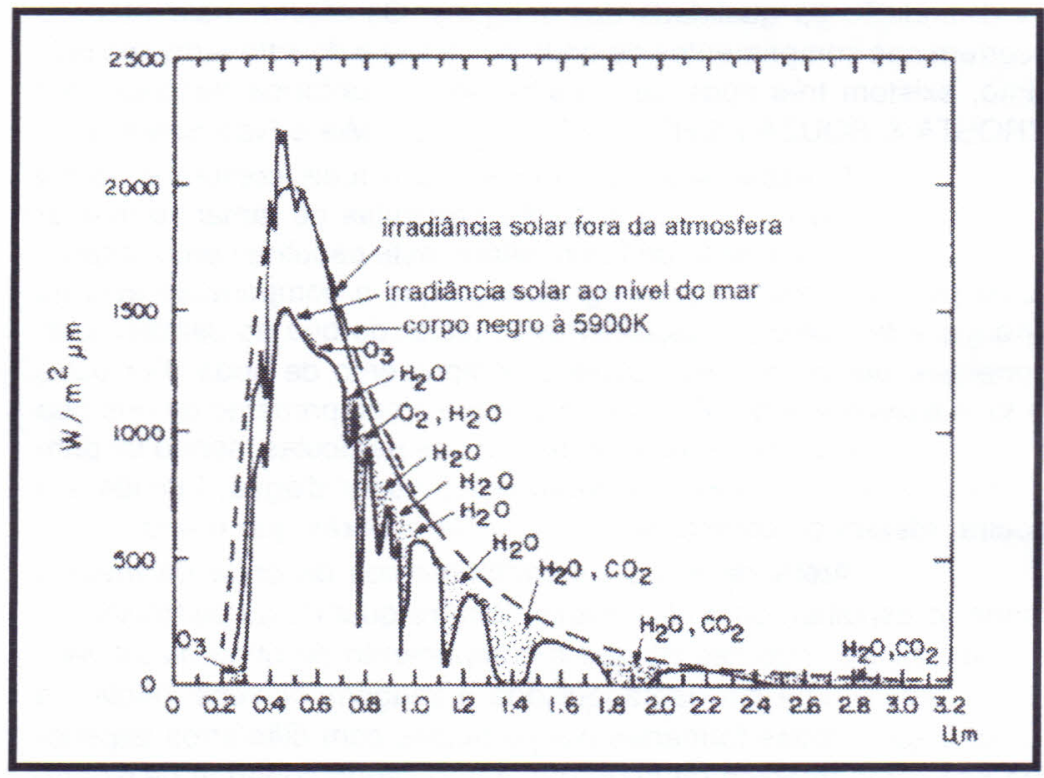

Fig. 1. Janelas atmosféricas e bandas de absorção. Fonte: Slater (1980) apud NOVO (1992).

A imagem gerada por um sensor remoto é constituída por uma matriz de pontos denominados pixel, onde cada um representa através de um número digital, a quantidade de radiação refletida ou emitida pela parte da cena que representa.

Os sensores captam informações resultantes da interação da energia eletromagnética ou radiação eletromagnética com objetos (alvos) da superfície terrestre, onde essa energia pode ser: refletida, transmitida, absorvida ou emitida pela superfície. 


\section{$E_{i}(\lambda)=E_{r}(\lambda)+E_{a}(\lambda)+E_{t}(\lambda)$}

A quantidade de energia que será refletida, absorvida ou transmitida depende das propriedades físico químicas, da estrutura e da posição do alvo em relação à fonte de radiação, além do comprimento de onda incidente (Novo, 1980, apud TODT 1998). Porém os objetos terrestres também emitem radiação eletromagnética cuja a quantidade e qualidade depende da natureza e da temperatura dos mesmos (ROSA, 1992 e STEFFEN, 1996).

Ao dividir-se cada um dos termos da equação anterior por $E_{i}(\lambda)$, se obtém a seguinte equação:

$$
1=\rho(\lambda)+\alpha(\lambda)+\tau(\lambda)
$$

Onde:

$$
\begin{aligned}
& \rho(\lambda)=\text { reflectância } \\
& \alpha(\lambda)=\text { absortância } \\
& \tau(\lambda)=\text { transmitância }
\end{aligned}
$$

Sabe-se que estes valores representam as porcentagens da energia incidente que é refletida, absorvida e transmitida pelo alvo, em um determinado comprimento de onda. A energia absorvida por um corpo ou é consumida para os processos (físicos/ biológicos - como a fotossíntese e a evapotranspiração), ou é liberada na forma de calor, sendo esta denominada de emitida. Para um alvo em estado de equilíbrio, é possível estabelecer a seguinte relação: $\varepsilon(\lambda) \cong \alpha(\lambda)$, teremos:

$$
1=\rho(\lambda)+\varepsilon(\lambda)+\tau(\lambda)
$$

Desta forma, na região termal do espectro eletromagnético, alvos com baixa reflectância possuem alta emitância, que é conseqüência de uma de uma alta absortância (válido para alvos que não possuem processos físicos/biológicos).

Conforme Fontana (1995) apud TODT (1998), assumese que os alvos são opacos à radiação, ou seja, a transmissividade é igual a zero, com exceção da água, assim teremos:

$$
1=\rho(\lambda)+\varepsilon(\lambda)
$$

Em sensoriamento remoto somente as chamadas janelas atmosféricas do espectro eletromagnético são utilizadas: visível, infravermelho próximo, infravermelho médio e infravermelho termal e microondas.

A captação e o registro pode ser feita por métodos analógicos (fotografia), onde a captação é feita pela câmara e o registro em filmes fotográficos, já os métodos eletrônicos exemplificase por uma câmara de vídeo. Os termos fotografia e imagem, são 
utilizados em sensoriamento remoto para distinguir as representações pictóricas geradas.

Para CROSTA \& SOUZA FILHO (1997), a aquisição de dados por sensores remotos obedece certos critérios que irão definir o seu conteúdo de informação, onde este critério refere-se à resolução, que pode ser subdividida em: resolução espacial, resolução espectral e resolução radiométrica.

Resolução é uma medida da habilidade que um sensor possui de distinguir entre respostas que são espectralmente ou próximas espacialmente (NOVO, 1989).

A capacidade que um sensor possui em registrar detaIhes espaciais da superfície é conhecida como resolução espacial. Quanto menor o objeto detectável, maior a resolução espacial. Em geral, a dimensão da menor célula no terreno, conhecida como pixel, é uma função da resolução espacial.

A resolução espectral é definida pelos intervalos do espectro eletromagnético cobertos pelo sensor, pelo número de bandas espectrais e pela largura espectral de cada banda. Quanto maior o número de bandas e mais estreita forem estas, maior a resolução espectral.

A quantidade de níveis de tons de cinza usados para expressar as variações na intensidade da energia coletada pelo sensor é denominada de resolução radiométrica. Quanto maior o número de níveis, maior a resolução. O número total de tons de cinza de uma imagem de Sensoriamento remoto, é geralmente definido como uma potência de 2 .

\subsection{CCD (Charge Coupled Device)}

Conforme ROSA (1992), o CCD é um conjunto de vários detetores, os quais utilizam-se da microeletrônica e o silício como material básico, juntos em um circuito integrado. Possui grande precisão geométrica, em conseqüência da não utilização de parte mecânica, sendo esta uma das vantagens do CCD. A alta sensibilidade, exige porém uma calibração dos detetores.

O CCD é um detector dinâmico, no estado sólido, baseado no processo de transferência coletivo de toda a carga elétrica móvel acumulada em um elemento semi condutor para seu vizinho através de tensões externas, possui cobertura espectral que vai do ultra violeta até cerca de 11.000 Ao $(1,1 \mu)$. Fabricado sobre uma 
base de silício e desenhado em esquema de circuito, e vaporizado com um filme condutor, os canais correspondentes as paredes, e estas são elétricas, sendo posteriormente limpas deste filme para que a corrente elétrica possa circular.

Cada elemento fotosensível do CCD é chamado de poço potencial, é aonde os elétrons são gerados e armazenados (paredes elétricas). A entidade física de um CCD é o diodo, podendo variar de tamanho, geralmente de $25 \mu$ a $30 \mu$. Existem CCDs com diodos de até $15 \mathrm{~m}$. O pixel é um elemento lógico da imagem, pode ser formado de um ou vários diodos, sendo essa quantidade dada por um tratamento chamado de Binning, o qual determina a resolução.

Cada diodo armazena no máximo 232 elétrons, acima ocorre saturação. Portanto, a capacidade de geração máxima de níveis de cinza é de 4,3 × 106 e um computador deve ter pelo menos uma capacidade de leitura de níveis de cinza próximo ao número gerado pelo CCD, para uma melhor interpretação da imagem. A saturação é causada pelo transbordamento dos elétrons do poço potencial de um diodo para poços vizinhos, ocorrendo em virtude do excesso de energia incidente.

A sensibilidade de cada diodo no CCD varia de um para outro na fase de fabricação, essa variação é da ordem de $1 \%$ a $3 \%$ na extensão do chip. Em termos de carga, a variação pode ser de $1 \%$ até $20 \%$, de um lado a outro do CCD. A solução neste caso é corrigir a inomogeneidade através da sensibilidade superficial, sendo feito com a técnica de medição do flat field. Isso implica na iluminação preliminar do chip com uma luz difusa e uniforme, medindo-se a sensibilidade do CCD e procedendo-se, posteriormente a correção da imagem gerada.

Quando uma série de diodos encontram-se desligados isso provoca um defeito na imagem, que aparece na forma de uma série de pixel não imageados (pixel mortos), não há correção possível para isso. Os chips no momento de fabricação, são classificados um a um, segundo sua homogeneidade. O tamanho dos chips, estão relacionados com o número de diodos $(800$ x 800, 1024 x 1024, etc.).

EI-HAKIM (1986), descreve que o CCD é composto de elementos distintos fotosensíveis (sensores) os quais podem ser semicondutores de metal-óxido (MOS) ou fotodioides. A energia que incide sobre cada elemento forma uma carga elétrica proporcional a intensidade de luz total. Essas cargas elétricas são coletadas em capacitores e então transferidas para um amplificador o qual apre- 
senta a imagem como uma série de voltagens, essas voltagens são quantidades analógicas, devem então ser convertidas em números separados, para processamento pelo computador, por meio da conversão analógica para digital.

A leitura em um CCD, ocorre através da incidência de fótons sobre o detector ocorrendo a liberação de elétrons em cada diodo. Os elétrons liberados permanecem em seus respectivos diodos e vão sendo acumulados durante o tempo de integração. Após a leitura (carga acoplada) ocorre a transferência coletiva de toda a carga de elétrons móveis armazenados num poço para um elemento vizinho sob a ação de tensões externas. Assim que diminui a diferença de potencial das paredes os elétrons se movem para poços vizinhos e todos os poços vão se esvaziando um a um, de acordo com um andamento pré-determinado e característico do "chip". Na saída o número de elétrons e dado sob a forma de matriz. O CCD é classificado como detector multicanal, porque as informações sobre a imagem se dão por blocos de informação, pois os sensores ou fotodetectores são os diodos, que estão dispostos em uma plataforma bidimensional, sendo assim esta a forma de imageamento.

\section{APLICAÇÕES}

Conforme BUNNIK et al. (1986), os detectores CCD são usados no Scanner Multiespectral Óptico-eletrônico Modular Alemão (MOMS) e nos instrumentos HRV do satélite francês SPOT. O scanner de alta resolução que usa os comprimentos de onda do visível e do infravermelho (VNIR) do satélite japonês MOS-1 também já faz parte operacional do uso de detectores CCD. Os autores afirmam que na utilização de um sistema aéreo as vantagens estão relacionadas ao dimensionamento compacto e a flexibilidade em relação a possibilidade de alteração dos filtros espectrais e na configuração para propósitos experimentais.

Nas instalações do Programa Nacional Holandês de sensoriamento remoto, um scanner multiespectral aéreo baseado no uso de detectores CCD foi desenvolvido e testado, o CAESAR: CCD Airborne Experimental Scanner for Applications in Remote Sensing. Os objetivos foram de obter experiência técnica com as propriedades eletro-ópticas dos CCDs, e propiciar o uso de um sistema aéreo versátil em programas experimentais de sensoriamento remoto. BUNNIK et al. (1986), relatam que o programa de demonstração será executado em cooperação com os usuários, testando e 
validando a capacidade para observações terrestres e marítimas, aplicações dos dados do CAESAR de alta resolução em combinação com dados de satélites de alta resolução disponíveis em uma técnica de vários estágios.

Os mesmos autores relatam que a calibração radiométrica relativa é necessária para a comparação quantitativa da correspondência de dados com uma imagem multiespectral única e com uma série temporal de imagens multiespectrais. No caso da calibração absoluta, a função de transferência é conhecida entre a radiância espectral no orifício de entrada de luminosidade do instrumento e do detector do sinal medido. A calibração absoluta é necessária apenas se o usuário quiser correções radiométricas por meio de dados externos (a partir de outros instrumentos ou modelos) e para o resgate de parâmetros do objeto a partir de valores de radiância fisicamente definidos.

A aquisição de imagens digitais, utilizando câmara de vídeo SONY CAMCORDER Handycam ( $8 \mathrm{~mm}$ ), possui CCD retangular de $315 \times 240$ pixeis, sendo esta equipada com filtros verde $(0,55$ $\mu \mathrm{m})$, vermelho $(0,65 \mu \mathrm{m})$ e infravermelho próximo $(0,72 \mu \mathrm{m})$ conforme determinação de espectro dos filtros, possibilitando assim a aquisição de imagens multiespectrais, sendo que com as mesmas é possivel a implementação de técnicas de processamento de imagens multiespectrais utilizando as imagens geradas pela câmara de vídeo (WATZLAWICK, 2000).

As imagens fotográficas digitais podem ser obtidas, utilizando-se câmaras de uso profissional, como por exemplo câmaras de médio formato que possuem maior precisão da parte ótica e apresentam diferentes dimensões físicas do CCD, os quais possuem precisão geométrica melhor, em conseqüência da não utilização de parte mecânica, sendo esta uma das vantagens da utilização do CCD, porem a alta sensibilidade exige uma calibração dos detetores.

\section{CONCLUSÕES}

A aplicação de detectores lineares direcionados para o imageamento multiespectral possui as seguintes vantagens: aumento do tempo de integração durante o qual a resolução espacial pode ser aumentada ou diminuída, melhora da imagem em termos de geometria devido a ausência de partes mecânicas móveis, em comparação com as técnicas clássicas de scaneamento mecânico. As desvantagens estão relacionadas ao aumento das exigências na qualidade 
da imagem no plano focal dos ópticos principais e da calibração radiométrica necessária para todos os elementos detectores individuais. Além disso para a maioria dos detectores CCD comercialmente disponíveis fabricados a partir de condutores de silício, a variação dos comprimentos de onda é limitada entre $0,4 \mathrm{~mm}$ e $1,1 \mathrm{~mm}$.

As câmaras que utilizam CCD são compatíveis com Hardware Digital e com as técnicas de processamento de imagens, também possuem as vantagens de serem pequenas, leves, aparentemente livres de manutenção, de vida longa e de alta resistência a descargas elétricas. O mais importante é que os sensores são estáveis ao longo do tempo, tanto em posição como em sensibilidade, com o aumento da resolução espacial das novas câmaras e pelas vantagens acima mencionadas, o CCD é o sensor escolhido para aplicações fotogramétricas, conforme afirma EL-HAKIM (1986). 


\section{REFERÊNCIAS BIBLIOGRÁFICAS}

BUNNIK, N. J. J.; POUWELS, H.; SMORENBURG, C.; VALKENBURG, A. L. G.van. CAESAR: CCD Airborne Experimental Scanner for Applications in Remote Sensing. In: SYMPOSIUM ON REMOTE SENSING FOR RESOUCES DEVELOPMENT AND ENVIRONMENTAL MANAGEMENT, Enschede, 1986, Proceedings..., p. 207- 210.

CROSTA, A. P., SOUZA FILHO, C. R. Sensoriamento remoto. In: ANUÁRIO FATOR GIS 1977. Curitiba: Sagres, 1977, 188p.

EL-HAKIM, S. F. Real Time Image Metrology with CCD Cameras. Photogrammetric Engeineering \& Remote Sensing, v. 52, No 11, November. 1986, p. 1757-1766.

NOVO, E. M. L. de. Sensoriamento remoto princípios e aplicações. São José dos Campos, SP: Edgard Blücher LTDA, 1989, $308 \mathrm{p}$.

NOVO, E. M. L. de. Sensoriamento remoto princípios e aplicações. São José dos Campos, SP: Edgard Blücher LTDA, 1992, $308 \mathrm{p}$.

ROSA, R. Introdução ao Sensoriamento Remoto. Uberlândia: Udufu, 1992, 117p.

STEFFEN, C. A. Radiometria Óptica Espectral - Fundamentos de Radiometria. SIMPÓSIO BRASILEIRO DE SENSORIAMENTO REMOTO, 8., 1996, São José dos Campos, SP. Tutorial. São José dos Campos: INPE. CD-ROM.

TODT, V. Avaliação do Desempenho de Classificadores Neurais para Aplicações em Sensoriamento Remoto. (Dissertação de Mestrado) CEPSRM - UFRGS. 1998, 232p.

WATZLAWICK, L. F. Avaliação de Classificadores a Partir de Dados Coletados Através de Videografia Multiespectral. (Dissertação de Mestrado) CEPSRM - UFRGS. 2000, 138p. 
LUCIANO Farinha WatZLAWICK luciano_farinha@uol.com.br Centro de Ciências Florestais e da Madeira Universidade Federal do Paraná Curitiba, PR - Brasil PedroRoberto de Azambuja Madruga madruga.sma@zas.com.br Rudiney Soares Pereira rudiney@ccr.ufsm.br Centro de Ciência Rurais - $\mathrm{Dpt}^{\circ}$. Eng. Rural Universidade Federal de Santa Maria Santa Maria, RS - Brasil 